\title{
Narrowing the blockade field: development of an optimal postoperative analgesia regimen for total knee arthroplasty
}

\author{
Ban C. H. Tsui, MD
}

Received: 18 January 2016/ Accepted: 8 February 2016/Published online: 17 February 2016

(C) Canadian Anesthesiologists' Society 2016

Total knee arthroplasty (TKA) is associated with significant postoperative pain. Pain management for TKA is complex, and even with administration of multiple nerve blocks, highquality postoperative analgesia cannot always be achieved. Multiple regional approaches, including femoral nerve block (FNB), fascia iliaca block, conventional or three-in-one (i.e., inguinal paravascular) lumbar plexus block, and adductor canal block (ACB) (with or without periarticular infiltration), have been attempted to minimize knee pain following the procedure with variable success. ${ }^{1,2}$ Historically, FNB has been the most commonly performed regional technique. Although FNB provides substantial coverage of the operative area, it may spare areas innervated by other inferior branches of the lumbar plexus, including the lateral femoral cutaneous and obturator nerves, resulting in inadequate analgesia. Since branches of the sciatic nerve also innervate the knee, various studies have examined whether supplementing FNB with sciatic nerve block (SNB) provides superior analgesia following TKA. In this issue, Abdallah et al. discuss their meta-analysis of several recent randomized-controlled trials, which they performed in an attempt to address the effectiveness of a combined FNB and SNB approach. ${ }^{3}$ The authors show that, although individual studies provide mixed results, overall trends suggest that combining SNB and FNB can reduce opioid consumption and alleviate knee pain postoperatively compared with FNB alone.

As Gaston Labat, the father of modern regional anesthesia, stated in his classic textbook, "Anatomy is

B. C. H. Tsui, MD ( $\square)$

Department of Anesthesiology and Pain Medicine, University

of Alberta, 2-150 Clinical Sciences Building, Edmonton,

AB T6G 2G3, Canada

e-mail: btsui@ualberta.ca the foundation upon which the entire concept of regional anesthesia is built." 4 Thus, a comprehensive understanding of anatomy is critical in order to select the appropriate block for the procedure and to provide effective analgesia. Branches from the lumbar (femoral and obturator nerves) and sacral (tibial and common peroneal nerves) plexuses provide innervation to the knee joint, and the saphenous nerve, a branch of the posterior division of the femoral nerve, provides some sensory supply to the medial aspect of the knee. The involvement of multiple nerves highlights the complexity of providing quality analgesia following knee surgery. In theory, a combination of lumbar plexus block and proximal SNB would provide the most effective analgesia; however, this approach would be complicated technically and would likely result in extensive motor block of the entire lower extremity. Although FNB is generally considered the gold standard for TKA analgesia, it has declined in popularity in favour of approaches that block a narrower region proximal to the knee. Indeed, one reason FNB has gradually fallen out of favour is its propensity for prolonged motor blockade. This attribute can cause quadriceps weakness and a delay in patient mobilization and rehabilitation, which raises concerns about delayed discharge from the hospital. Since proximal SNBs (which were the only approaches included in the Abdallah et al. meta-analysis) target the sciatic nerve before its bifurcation, both the tibial and common peroneal nerves are anesthetized, increasing the potential for sensory and motor blockade. Nevertheless, Abdallah et al. found that the addition of SNB did not significantly delay functional recovery or discharge from hospital. ${ }^{3}$ This finding suggests that the FNB alone can be responsible for the motor impairment that precludes early mobilization and that its effects eclipse any effects of the SNB that would impede early mobilization. 
The unwanted motor blockade associated with FNB has resulted in ACB gaining favour among regional anesthesiologists. This became particularly apparent following the expansion of the use of ultrasound, which has made it relatively easy to identify the site for the injection of a minimal amount of local anesthetic to achieve a good block. ${ }^{5}$ The adductor (subsartorial or Hunter's) canal runs posterior to the sartorius muscle and extends from the femoral triangle to the adductor hiatus. In addition to housing the femoral artery and vein, the canal also contains the nerve to the vastus medialis muscle, the saphenous nerve, and the posterior division of the obturator nerve. ${ }^{6}$ As such, effective sensory blockade of the knee joint can be achieved while theoretically sparing blockade of motor fibres supplying the quadriceps muscles. This results in shortened time to mobilization compared with conventional FNB. Two recent meta-analyses suggest that ACB offers analgesia that is comparable, if not superior, to FNB while enabling earlier ambulation and functional recovery. ${ }^{7,8}$ When used in addition to periarticular local infiltration, ACB provides quality analgesia and rapid ambulation and home discharge. ${ }^{9}$ Nevertheless, ACB is not without its shortcomings; indeed, reports of quadriceps weakness $^{10}$ and unwanted partial $\mathrm{SNB}^{11}$ have been published. In addition, ACB must be performed with care since the injection site is close to major vessels (increasing the risk of systemic toxicity). At the same time, variability in sensory nerve anatomy within the adductor canal means that blockade of all necessary nerves may not always occur. Paradoxically, ACB provides good analgesia for TKA yet does not target sensory fibres of the sciatic nerve and its branches. This leads us back to the issue addressed by Abdallah et al. -i.e., whether additional SNB is in fact necessary for pain control following TKA.

The tibial nerve provides most of the innervation to the posterior knee, so it stands to reason that SNB would provide sufficient pain control to this part of the joint. The evidence in favour of SNB for TKA analgesia is relatively strong (grade $\mathrm{A}^{12}$ recommendation), although the considerable heterogeneity among the studies analyzed by Abdallah et al. limits the strength of their analysis. ${ }^{3}$ Moreover, as acknowledged in the review, most individuals have trouble discerning pain in regions of the knee supplied by different nerves (e.g., femoral for the anterior knee, sciatic for the posterior) since the nerves have overlapping distributions in this area. Nevertheless, we must remain cognizant of the fact that, along with offering supplemental analgesia, SNB may produce significant weakness ${ }^{1}$ of the lower calf and/or cause foot drop, further complicating the goal of early rehabilitation.

Major nerves like the sciatic and femoral nerves generally provide sensory supply in addition to controlling motor activity. Unless a "magic" local anesthetic is available that, when applied to the same nerve, targets only the sensory supply and spares the motor activity completely, we must achieve a balance between maximal sensory block and minimal motor block. For the last few decades, regional analgesia for TKA (or in general) has involved working towards narrowing the fields of blockade - i.e., from central neuraxial to individual major nerve(s) to distal individual nerves. Without question, improvements in technology primarily ultrasonography - have increased our knowledge and ability to visualize and block major nerves accurately. The next challenge is toward further improvement in technology or technique to allow blockade of smaller nerves in a more defined target area. Therefore, it falls upon experts in regional anesthesia to push the envelope and continue to develop techniques that allow for more precise targeting of nerve blocks.

At first glance, the article by Abdallah et al. may appear contrary to current trends since it does not specifically address popular methods of TKA analgesia such as ACB or local infiltration. Nevertheless, the authors should be commended for their painstaking and well-written metaanalysis, as they do well to raise a fundamental question i.e., whether SNB is a useful adjunct to FNB for pain control following TKA. In particular, coverage of the posterior knee has been a troublesome issue in regional anesthesia for TKA, especially if one is using the currently popular ACB. Although addition of SNB is one solution, in this context, it is akin to using a sledgehammer to crack a nut because of the associated motor blockade and delays in mobilization and rehabilitation. Thus, the focus of future work must be on discovery or development of more direct blocks such as local infiltration or multimodal regimens that spare the knee's motor supply as much as possible while providing sufficient analgesia to all aspects of the joint. As with any advancement in regional anesthesia, this work will rely on sound anatomical knowledge, appropriate technology, and the ambition to refine current techniques safely and creatively to suit our needs and those of our patients.

\section{Comment réduire le champ du bloc: mise au point d'un régime d'analgésie postopératoire optimal pour l'arthroplastie totale du genou}

L'arthroplastie totale du genou (ATG) est associée à une douleur postopératoire considérable. La prise en charge de la douleur pour l'ATG est complexe et, même en réalisant plusieurs blocs nerveux, on ne parvient pas toujours à 
obtenir une analgésie postopératoire de bonne qualité. Plusieurs approches régionales, y compris le bloc du nerf fémoral (BNF), le bloc ilio-fascial, le bloc du plexus lombaire conventionnel ou trois-en-un (c.-à-d. inguinal paravasculaire) et le bloc du canal des adducteurs (BCA) (avec ou sans infiltration périarticulaire), ont été utilisées pour tenter de minimiser la douleur au genou après l'intervention et ce, avec un succès variable. ${ }^{1,2} \mathrm{La}$ technique régionale la plus fréquemment réalisée est incontestablement le BNF. Bien que la zone opératoire couverte par le BNF soit considérable, il arrive néanmoins que ce bloc épargne certaines zones innervées par d'autres branches inférieures du plexus lombaire, notamment les nerfs cutanés et obturateurs fémoraux latéraux, ce qui pourrait empêcher une analgésie optimale. Étant donné que certaines branches du nerf sciatique innervent également le genou, plusieurs études ont tenté de déterminer si l'ajout d'un bloc du nerf sciatique (BNS) au BNF procurerait une meilleure analgésie après une ATG. Dans ce numéro du Journal, Abdallah et coll. présentent leur méta-analyse, qui porte sur plusieurs études randomisées contrôlées récentes. Leur objectif était d'examiner l'efficacité d'une approche combinant BNF et BNS. ${ }^{3}$ Les auteurs montrent que, bien que les études individuelles présentent des résultats mitigés, les tendances globales laissent à penser que la combinaison de ces deux blocs pourrait réduire la consommation d'opioïdes et soulager la douleur postopératoire au genou, comparativement à une technique de BNF seul.

Comme le déclarait Gaston Labat, le père de l'anesthésie régionale moderne, dans son célèbre manuel, «L'anatomie est la fondation sur laquelle le concept entier d'anesthésie régionale est bâti. » ${ }^{4}$ Ainsi, une compréhension exhaustive et minutieuse de l'anatomie est absolument essentielle si l'on veut choisir le bloc adapté pour l'intervention et procurer une analgésie efficace. Des branches des plexus lombaire (nerfs fémoral et obturateur) et sacral (nerfs tibial et péronier commun) innervent l'articulation du genou et le nerf saphène, une branche de la division postérieure du nerf fémoral, est responsable de l'innervation du côté interne du genou. L'implication de nombreux nerfs ne fait que souligner la complexité d'une analgésie de qualité après une chirurgie du genou. En théorie, la combinaison d'un bloc du plexus lombaire et d'un BNS proximal devrait procurer l'analgésie la plus efficace; toutefois, cette approche comporte des difficultés techniques et résulterait probablement en un bloc moteur étendu du membre inférieur dans son intégralité. Bien que le BNF soit, en règle générale, considéré la norme d'excellence de l'analgésie pour l'ATG, sa popularité a décliné en faveur d'approches qui bloquent une région plus petite et plus proche du genou. En effet, une des raisons pour lesquelles le BNF est progressivement tombé en disgrâce réside dans sa propension à créer un bloc moteur prolongé. Cette particularité peut entraîner une faiblesse au niveau du quadriceps et un retard dans la mobilisation du patient et sa récupération - découlant donc en inquiétude quant à un possible retard dans le congé de l'hôpital. Étant donné que les BNS proximaux (les seules approches incluses dans la méta-analyse d'Abdallah et coll.) ciblent le nerf sciatique avant sa bifurcation, les nerfs tibial et péronier commun sont tous deux anesthésiés, ce qui augmente le risque de bloc sensitif et moteur. Toutefois, Abdallah et coll. ont découvert que l'ajout d'un BNS ne retardait pas de façon significative la récupération fonctionnelle ou le congé de l'hôpital. ${ }^{3}$ Cette observation laisse à penser que le BNF seul pourrait être responsable de la perte de motricité qui empêche une mobilisation précoce et que ces effets éclipsent tout effet du BNS qui pourrait retarder une mobilisation précoce.

En raison du bloc moteur indésirable associé au BNF, le BCA a gagné en popularité parmi les anesthésiologistes régionaux. Cela est devenu particulièrement flagrant à la suite de l'essor de l'utilisation de l'échoguidage, qui rend relativement facile l'identification du site d'injection d'une quantité minimale d'anesthésique local pour établir un bloc efficace. ${ }^{5}$ Le canal des adducteurs (ou canal de Hunter) est postérieur au muscle sartorius et s'étend du triangle de Scarpa à l'hiatus de l'adducteur. Outre le fait qu'il loge l'artère et la veine fémorale, ce canal contient également le nerf du muscle vaste interne, le nerf saphène, et la portion postérieure du nerf obturateur. ${ }^{6}$ Dès lors, en théorie du moins, un bloc sensitif efficace de l'articulation du genou peut être réalisé tout en épargnant le bloc des fibres motrices qui alimentent le quadriceps. Ainsi, le délai jusqu'à la mobilisation est plus court par rapport à un BNF conventionnel. Deux méta-analyses récentes suggèrent que le BCA procure une analgésie comparable, voire supérieure, à un BNF, tout en favorisant une ambulation et une récupération fonctionnelle plus précoces. ${ }^{7,8}$ Lorsqu'il est ajouté à une infiltration locale périarticulaire, le BCA procure une analgésie de qualité tout en favorisant une ambulation et un congé rapides. ${ }^{9}$ Ceci étant dit, le BCA n'est pas sans défaut; en effet, certaines publications font état d'une faiblesse au niveau du quadriceps ${ }^{10}$ ainsi que de BNS partiel non voulu. ${ }^{11}$ En outre, le BCA doit être réalisé avec soin étant donné que le site d'injection est proche de vaisseaux majeurs, ce qui augmente le risque de toxicité systémique. Dans le même temps, en raison de la variabilité en matière d'anatomie des nerfs sensitifs au sein du canal des adducteurs, le bloc pourrait ne pas toujours atteindre tous les nerfs souhaités. Paradoxalement, le BCA procure une bonne analgésie pour l'ATG mais ne cible pas les fibres sensitives du nerf sciatique, ni ses branches. Ainsi, retournons à la problématique à laquelle Abdallah et coll. se sont intéressés : un BNS supplémentaire est-il en fait nécessaire pour contrôler la douleur après une ATG? 
Le nerf tibial est responsable de la plus grande partie de l'innervation du genou postérieur. Par conséquent, il serait logique qu'un BNS procure un contrôle de la douleur suffisant de cette partie de l'articulation. Les données probantes appuyant le BNS pour l'analgésie de l'ATG sont relativement cohérentes (recommandation de grade A), ${ }^{12}$ bien que l'hétérogénéité considérable des études analysées par Abdallah et coll. limite quelque peu l'autorité de leur analyse. ${ }^{3}$ De plus, comme leur compte rendu le reconnaît, la plupart des personnes éprouvent de la difficulté à discerner une douleur dans les régions du genou innervées par différents nerfs (par ex., fémoral pour le genou antérieur, sciatique pour le postérieur) étant donné que les distributions nerveuses se chevauchent dans cette région. Toutefois, nous devons garder à l'esprit que, outre le fait d'offrir une analgésie supplémentaire, le BNS pourrait provoquer une faiblesse significative au niveau du mollet inférieur et/ou causer un pied tombant, ce qui compliquera davantage l'objectif de récupération précoce.

En règle générale les grands nerfs, tels que les nerfs sciatiques et fémoraux, sont aussi responsables de l'afférent sensitif, en plus de contrôler l'activité motrice. À moins qu'un anesthésique local « magique » ne soit disponible qui, lorsqu'il est appliqué au même nerf, ne cible que l'afférent sensitif et épargne totalement l'activité motrice, nous devons trouver un équilibre entre un bloc sensitif maximal et un bloc moteur minimal. Au cours des dernières décennies, l'analgésie régionale pour l'ATG (ou en général) s'est efforcée de réduire les champs du bloc - en passant d'une analgésie centrale neuraxiale, à une anesthésie des nerfs majeurs, puis des nerfs distaux individuels. Il ne fait aucun doute que les progrès technologiques, l'échographie en tête, ont augmenté nos connaissances et notre capacité à visualiser et bloquer les principaux nerfs de façon précise. Le prochain défi sera d'améliorer encore la technologie ou la technique afin de pouvoir bloquer des nerfs plus petits dans une zone cible mieux définie. Ainsi, il incombe aux experts de l'anesthésie régionale de repousser les limites et de continuer à mettre au point des techniques permettant de cibler de façon plus précise les blocs nerveux.

De prime abord, l'article d'Abdallah et coll. semble aller à contre-courant des tendances actuelles : en effet, il n'aborde pas spécifiquement les méthodes les plus populaires pour l'analgésie pour l'ATG, telles que le BCA ou l'infiltration locale. Toutefois, il faut féliciter les auteurs pour leur méta-analyse minutieuse et bien écrite, car ils font bien de soulever une question fondamentale, à savoir si le BNS est un ajout utile au BNF pour contrôler la douleur après une ATG. D'autant plus que la couverture du genou postérieur est problématique en anesthésie régionale pour l'ATG, particulièrement si l'on utilise la technique actuellement en vogue, soit le BCA. Bien que l'ajout d'un
BNS soit une solution, dans ce contexte, elle s'apparente à l'utilisation d'une massue pour ouvrir une noix en raison du bloc moteur associé et des retards dans la mobilisation et la récupération du patient. Pour cette raison, les travaux futurs devront se concentrer sur la découverte ou la mise au point de blocs plus ciblés tels que l'infiltration locale ou les régimes multimodaux qui épargnent la fonction motrice du genou autant que possible tout en procurant une analgésie suffisante à toutes les régions de l'articulation. Tout comme c'est le cas pour quelque progrès que ce soit en anesthésie régionale, ces travaux devront s'appuyer sur des connaissances anatomiques approfondies, des technologies adaptées, et l'ambition de peaufiner les techniques actuelles de façon sécuritaire et créative afin de répondre à nos besoins et à ceux de nos patients.

Acknowledgment Dr. Tsui is supported by a Clinical Scholar Award from the Alberta Heritage Foundation for Medical Research (AHFMR).

Le Dr Tsui est soutenu financièrement par une Bourse de chercheur-boursier clinique de la Fondation albertaine pour la recherche médicale (Alberta Heritage Foundation for Medical Research - AHFMR).

Conflicts of interest None declared.

Conflit d'intérêt Aucun.

\section{References}

1. Bauer MC, Pogatzki-Zahn EM, Zahn PK. Regional analgesia techniques for total knee replacement. Curr Opin Anaesthesiol 2014; 27: 501-6.

2. Danninger T, Opperer M, Memtsoudis SG. Perioperative pain control after total knee arthroplasty: an evidence based review of the role of peripheral nerve blocks. World J Orthop 2014; 5: 225-32.

3. Abdallah FW, Madjdpour C, Brull $R$. Is sciatic nerve block advantageous when combined with femoral nerve block for postoperative analgesia following total knee arthroplasty? a meta-analysis. Can J Anesth 2016; 63. DOI:10.1007/s12630-0160613-2.

4. Labat G. Regional Anesthesia: Its Technic and Clinical Application. Philadelphia: WB Saunders; 1922.

5. Manickam B, Perlas A, Duggan E, Brull R, Chan VW, Ramlogan $R$. Feasibility and efficacy of ultrasound-guided block of the saphenous nerve in the adductor canal. Reg Anesth Pain Med 2009; 34: 578-80.

6. Head SJ, Leung RC, Hackman GP, Seib R, Rondi K, Schwarz SK. Ultrasound-guided saphenous nerve block - within versus distal to the adductor canal: a proof-of-principle randomized trial. Can J Anesth 2015; 62: 37-44.

7. Li D, Ma GG. Analgesic efficacy and quadriceps strength of adductor canal block versus femoral nerve block following total knee arthroplasty. Knee Surg Sports Traumatol Arthrosc 2015. DOI:10.1007/s00167-015-3874-3.

8. Li D, Yang Z, Xie X, Zhao J, Kang P. Adductor canal block provides better performance after total knee arthroplasty compared with femoral nerve block: a systematic review and meta-analysis. Int Orthop 2015. DOI:10.1007/s00264-015-2998-x. 
9. Perlas A, Kirkham KR, Billing R, et al. The impact of analgesic modality on early ambulation following total knee arthroplasty. Reg Anesth Pain Med 2013; 38: 334-9.

10. Chen J, Lesser JB, Hadzic A, Reiss W, Resta-Flarer F. Adductor canal block can result in motor block of the quadriceps muscle. Reg Anesth Pain Med 2014; 39: 170-1.

11. Gautier PE, Lecoq JP, Vandepitte C, Harstein G, Brichant JF. Impairment of sciatic nerve function during adductor canal block. Reg Anesth Pain Med 2015; 40: 85-9.
12. Anderson JL, Adams CD, Antman EM, 2011 Writing Group Members, ACCF/AHA Task Force Members, et al. 2011 ACCF/ AHA Focused Update Incorporated Into the ACC/AHA 2007 Guidelines for the Management of Patients With Unstable Angina/ Non-ST-Elevation Myocardial Infarction: a report of the American College of Cardiology Foundation/American Heart Association Task Force on Practice Guidelines. Circulation 2011; 123: e426-579. 